\title{
UN THEOREME SUR LES GROUPES D'HOLONOMIE*
}

\author{
KATSUMI NOMIZU
}

1. MM. Ozeki et Hano ont récemment démontré [3] que tout sous-groupe connexe de Lie du groupe linéaire $G L(n, R), n \geqslant 2$, peut etre réalisé comme groupe d'holonomie d'une certaine connexion linéaire dans un espace affine de dimension $n$.

On se propose ici de donner une généralisation de ce résultat au cas de connexions infinitésimales dans une variété fibrée différentiable. On établit:

THÉorème. Soit $P(B, G)$ une variété fibrée principale à base $B$ de dimension $\geq 2$ et à groupe structural de Lie $G$. Si le groupe strustural $G$ peut être réduit à un sous-groupe connexe de Lie $H$, alors il existe dans $P$ une connexion infinitésimale dont le groupe d'holonomie restreint est exactement $H$ (au choix convenable d'un point de reférence dans $P$ ).

Ce théorème complète aussi le résultat suivant: si $H$ est le groupe d'holonomie d'une connexion infinitésimale dans $P(B, G)$, alors le groupe structural $G$ peut être réduit à $H$ (Proposition, p. 37 [1], voir aussi [2]).

Corollaire. Tout groupe connexe de Lie G peut être réalisé comme groupe d'holonomie restreini d'une certaine connexion infinitésimale dans une variété fibrée principale à base variété quelconque de dimension $\gtrsim 2$.

En effet, étant donnée une variété différentiable quelconque $B$, il suffit de considérer la variété fibrée principale $P=B \times G$, produit direct.

2. Avant d'entrer dans la démonstration du théorème, il est utile de faire deux remarques suivantes sur les connexions infinitésimales dans une variété fibrée principale $P(B, G)$.

Réduction du groupe structural. On dit que le groupe structural $G$ peut être réduit à un sous-groupe de Lie $H$, s'il existe une variété fibrée principale

Received December 20, 1955.

* Ce travail a été accompli pendant que l'auteur était boursier de la Fondation Yukawa. 
$Q(B, H)$, à base $B$ et à groupe structural $H$, munie d'un isomorphisme différentiable $f$ de $Q$ dans $P$, c'est-à-dire, une application différentiable biunivoque $f$ de $Q$ dans $P$ telle que l'on ait $f(x a)=f(x) a$ et $\pi(f(x))=\pi(f(x))$ pour tout $x \in B$ et pour tout $a \in H$ ( $\pi$ désignant la projection canonique de $Q$ sur $B$ et celle de $P$ sur $B$ ).

On sait [2] que, dans ce cas-là, une connexion infinitésimale quelconque $r$ dans $Q$ induit une connexion infinitésimale dans $P$ dont le groupe d'holonomie est égal à celui de $\Gamma$.

Prolongement d'une connexion infinitésimale locale. Soit $U$ un ouvert de $B$. Une connexion infinitésimale locale sur $U$ est, par définition, une connexion infinitésimale dans $\pi^{-1}(U)$, partie de $P$ sur $U$, qui est une variété fibrée principale d'une façon naturelle.

En tenant compte de la démonstration (p. 40, [1]) de l'existence de connexions infinitésimales et en utilisant le théorème bien connu (p. 25, [4]) sur le prolongement de sections locales dans une variété fibrée différentiable dont la fibre est homéomorphe à un espace numérique, on obtiendra: Soient $U$ et $V$ deux ouverts de $B$ tels que $\bar{V} \subset U$. Etant donnée une connexion infinitésimale locale $\Gamma$ sur $U$, il existe dans $P$ une connexion infinitesimale qui coïncide avec $\Gamma$ dans $\pi^{-1}(V)$.

3. La démonstration du théorème s'achève comme suit. En vertu de la première remarque dans 2 , il suffit d'établir l'existence d'une connexion infinitésimale dans $P$ dont le groupe d'holonomie restreint est égal à $G$.

Pour cela, soit $U$ un ouvert de $B$ tel que $\pi^{-1}(U)$ soit isomorphe au produit direct $U \times G$. On supposera que $U$ est un voisinage d'un point $p$ avec un système de coordonnées $x^{1}, x^{2}, \ldots, x^{n}, n \geqslant 2$, pour lesquelles $U=\left\{\left(x^{i}\right) ;\left|x^{i}\right|<\alpha\right\}, \alpha>0$, et $x^{i}(p)=0, i=1,2, \ldots, n$. Soit $V=\left\{\left(x^{i}\right) ;\left|x^{i}\right|<\beta\right\}$, où $0<\beta<\alpha$. On va construire une connexion infinitésimale locale $\Gamma$ sur $U$ telle que le groupe d'holonomie de la connexion induite sur $V$ soit égal à $G$; cela fait, on n'a qu'à prolonger $\Gamma$ de la manière indiquée dans 2.

Enfin, on se trouve dans le cas suivant: $P$ est le produit direct $U \times G$, où $U=\left\{\left(x^{i}\right) ;\left|x^{i}\right|<\alpha\right\}$. Pour $V=\left\{\left(x^{i}\right) ;\left|x^{i}\right|<\beta\right\}$, où $\beta<\alpha$, il s'agit de construire une connexion infinitésimale dans $P$ telle que le groupe d'holonomie de la connexion induite dans $V \times G$ soit égal à $G$. C'est là la question essentiellement 
résolue dans [3]. Soit $\mathrm{g}$ l'algèbre de Lie du groupe $G$ et soit $\left\{A_{1}, A_{2}, \ldots, A_{r}\right\}$ une base de $\mathfrak{g}$. Pour $r$ nombres $a_{1}, \ldots, a_{r}$ tels que $0<a_{1}<a_{2}<\ldots<a_{r}<\beta$, soient $f_{i}(t), i=1, \ldots, r$, des fonctions différentiables dans $-\alpha-\varepsilon<t<\alpha+\varepsilon$, $\varepsilon>0$, qui satisfont aux conditions

$$
d f_{i} / d t\left\{\begin{array}{l}
=0 \text { pour } t=a_{j}, \quad j \neq i \\
=c_{i} \neq 0 \text { pour } t=a_{i} .
\end{array}\right.
$$

On définit une connexion infinitésimale dans $P=U \times G$ au moyen de la forme de connexion (1) qui est complètement déterminée par les conditions suivantes:

$$
\begin{aligned}
& \omega_{(x, e)}\left(X_{1}\right)=\sum_{k=1}^{n} f_{k}\left(x^{2}\right) A_{k} \\
& \omega_{(x, e)}\left(X_{i}\right)=0, \quad i=2, \ldots, n,
\end{aligned}
$$

où $X_{i}$ sont les champs de vecteurs sur $U \times G$ qui sont la différentiation partielle par rapport d̀ $x^{i}, i=1,2, \ldots, n$.

En choisissant le point $(p, e) \in V \in G, p=(0,0, \ldots, 0)$, comme point de référence, on voit, tout à fait à la même manière que [3], que le groupe d'holonomie de la connexion induite sur $V \times G$ est égal à $G$.

\section{BiBLIOGRAPHIE}

[1] C. Ehresmann, Les connexions infinitésimales dans un espace fibré différentiable, Colloque de Topologie, Bruxelles, 1950.

[2] K. Nomizu, Reduction theorem for connections and its application to the problem of isotropy and holonomy groups of a Riemannian manifold, Nagoya Math. Journ. 9 (1955), pp. 57-66.

[3] H. Ozeki et J. Hano, On the holonomy groups of linear connections, à ce journal.

[4] N. Steenrod, The topology of fiber bundles, Princeton, 1951.

Institut de Mathématiques

Universite de Nagoya 